\title{
Immunohistochemical Characterization and Subclassification of Triple Negative Breast Cancer of Egyptian Female Patients
}

\author{
AL-SHIMAA ZAKARIA, M.Sc. ${ }^{\text {; }}$ NAGLAA EL-KINAAI, M.D. ${ }^{2}$; IMAN LOAY, M.D. ${ }^{\text {; }}$; \\ HANAN R. NASSAR, M.D. ${ }^{3}$; TAREK DARWISH, M.D. ${ }^{3}$; MERVAT EL-DEFTAR, M.D. ${ }^{1}$; \\ RASHA M. ALLAM, M.D. ${ }^{4}$ and HASAN NABIL, M.D. ${ }^{1}$ \\ The Department of Pathology, National Cancer Institute 1 , Department of Pathology, National Cancer Institute \& Children's \\ Cancer Hospital ${ }^{2}$, Department of Medical Oncology, National Cancer Institute 3 and Department ofBiostatistics \& Cancer \\ Epidemiology, National Cancer Institute4, Cairo University, Egypt
}

\begin{abstract}
Background: Triple negative breast cancer is representing a heterogeneous group of breast cancer. Many trials were carried out for its subclassification by different markers. This classification also could help in new treatment modalities.

Aim of Study: To investigate and identify additional possible prognostic markers that can help in subclassification of triple negative breast cancer.

Patients and Methods: This retrospective study included 59 women with triple negative breast cancer referred to NCI, Cairo University, Egypt. Clinical data were collected. Immunohistochemical analyses were performed for the assessment of CK5/6, Epithelial growth factor receptor (EGFR), and Claudin 4. All cases were followed-up with a median followup time 40.5 months (range: 8-67).

Results: Among the studied cases $(71.2 \%)$ were positive to $\mathrm{CK} 5 / 6,(45.8 \%)$ were positive to EGFR, $(89.8 \%)$ represented Claudin 4 high. Expression of CK5/6 revealed a significant relationship with tumor size. Expression of EGFR revealed a significant relationship with stage of the tumor. Most of cases of Claudin 4 high represented high grade, high stage and node positivity, but not reached the significant level. There is significant relationship found between grade and DFS, as well as OS with grade, stage, occurrence of metastasis and node positivity.

Conclusion: Triple negative breast cancer is a heterogeneous molecular type of breast cancer. Basal markers such as CK5/6 and EGFR are used for identification of basal subgroup of TNBC. This subclassification of triple negative breast cancer helps in categorization of patients who are candidate for targeted therapy, such as tyrosine kinase inhibitors (TKIs) in cases positive for EGFR. Expression of Claudin 4 also helps in classification of triple negative breast cancer, and selection of cases that could be targeted by monoclonal antibodies.
\end{abstract}

Key Words: TNBC-EGFR - CK5/6 - Claudin 4.

\section{Introduction}

IN USA by the end of 2017, an estimated 252,710 new cases of invasive breast cancer were diagnosed

Correspondence to: Dr. Al-Shimaa Zakaria, The Department of Pathology, National Cancer Institute Cairo University, Egypt among women. Approximately 40,610 women died from breast cancer in 2017 [1]

In the National Cancer Institute, Cairo University (NCI-CU), breast cancer came as number one in ranking malignant tumors constituting $19.3 \%$ of total primary malignancies [2].

Previously the breast cancer classification was based on histological type as well as clinical and pathological parameters of the tumor (tumor size, grade, stage, and lymph node status) which was affecting treatment protocol [3]. The advances in breast cancer classification into molecular types represented a great discovery with marvelous effect on treatment strategy and prediction of prognosis [4].

The triple-negative breast cancer (TNBC) subtype accounts for approximately $15 \%$ of all breast cancers, and is defined pathologically by the absence of expression of the estrogen receptor (ER) and progesterone receptor (PR) and lack of over expression or amplification of the HER2/neu oncogene $[\mathbf{5 , 6 , 7 ]}$

According to (NCI-CU) cancer 12-years registry; TNBC constitutes about $13.5 \%$ of breast cancer [2]. Triple negative breast cancer is the most serious group molecular subtypes of breast cancer as it carries bad prognosis, early recurrence and metastasis [8]

As chemotherapy is currently the only treatment option for patients with triple negative breast cancer. Attempts for investigating the expression of various immunohistochemical markers which could aid in further subclassification of TNBC and providing new insights for treatment options are on the run $[\mathbf{9 , 1 0 ]}$

Such studies are actually lacking among Egyptian patients due to queries about coast effectiveness of such studies, their impact on the treatment 
options, and the availability of the targeted therapy against these antibodies.

Basal markers such as CK5/6 and EGFR are considered important in subclasssification of the triple negative group and can also affect treatment options.

CK5/6 is a high molecular weight cytokeratin that is frequently applied in the panel of markers used to differentiate benign and malignant lesions of the breast [11] it identifies basal like phenotype in up to $81 \%$ of invasive breast carcinomas by using immunohistochemistry [12] .

HER1/ EGFR is a receptor tyrosine kinase (RTK) that belongs to the ErbB family, and a transmembrane protein comprising an extracellular ligand binding domain, transmembrane domain, and cytoplasmic tyrosine kinase domain and plays an important role in cell proliferation, migration and protection against apoptosis. HER 1 protein could be targeted by monoclonal antibodies and/or tyrosine kinase inhibitors (TKIs). Given signal pathway complexity and HER family member cooperation, it may be better to simultaneously target a number of these receptors [9]

Claudin 4 is one of the transmembrane proteins which is important in tight junction of cells underwent many researches with great expectation to be targeted by targeting antibodies which used in designing new treatment planes [10]

The current study aimed to study the immunohistochemical expression of basal markers including CK5/6 and EGFR as well as Claudin 4 among triple negative breast cancer cases.

\section{Patients and Methods}

This retrospective study was carried out at the National Cancer Institute (NCI), Cairo University, Egypt, during the period from 2011 to 2014, diagnosed at pathology department. The study was approved by the Institutional Review Board of NCI. Written informed consent was obtained from all participants. The study included 59 women with triple negative breast cancer, which represented all case that met the inclusion criteria which were cases with available blocks, available follow-up with minimum time of two years. We excluded cases with lost files, unavailable blocks, incomplete follow-up data, and small non representative tumor samples.

Data of studied cases were collected from medical records in the pathology department as well as surgical oncology, medical oncology and biostatistics department. These included personal data, clinical data, and pathologic data. All studied cases received the same anthracyclin based chemotherapy.

Hematoxylin and Eosin slides were reviewed for histologic type according to the criteria mentioned by World Health Organization (WHO) [13] histological grade which evaluated using the Nottingham-combined histologic grade [Elston-Ellis modification of Scarff-Bloom-Richardson (SBR) grading system] [14]. Staging of tumors was carried out according to the American Joint Committee on Cancer (AJCC) TNM staging system of breast cancer, 7 th edition [15].

\section{Immunohistochemical methods.}

All immunohistochemical analyses were performed on routinely processed, formalin-fixed, paraffin-embedded tissues. Antibodies used were: Primary monoclonal antibodies against CK5/6 (Dako, mouse monoclonal, clone D5/16B4, ready to use), EGFR (Cell Marque, Rabbit monoclonal, clone SP84, ready to use) and Claudin 4 (Thermo fisher, mouse monoclonal,clone $3 \mathrm{E} 2 \mathrm{C} 1$, ready to use ). Three unstained sections were prepared on positively charged slides for the assessment of CK5/6, EGFR, and Claudin 4.

Positive control slides were used within each batch of slides (surface squamous epithelium of skin piece for CK5/6 as well as EGFR, and section of ovarian carcinoma for Claudin 4), the thickness of paraffin sections were made 4 microns and mounted on positive charged slides, then immunostaining was done using BenchMark XT (Ventana) autostainer following protocols instruction.

\section{Assessment of immunostaing:}

CK5/6: Immunoreaction was positive when brown, homogenous cytoplasmic or membranous staining of tumor cells was seen. Semiquantitative method were used including, 0: For cases with no immunostaining, 1+: For positive immunostaining in less than $10 \%, 2+$ : For positive immunostaining in $10 \%$ to $50 \%$, and $3+$ : For strongly positive immunostaining in $>50 \%$.

The scores 0 and 1 were considered as negative while scores 2 (Fig. 1A) and score 3 (Fig. 1B) were considered as positive [16].

EGFR: Clear staining of the tumor cell membranes was scored positive. Semiquantitative approach was used to generate a score for each tumor tissue sample: The percentage of positive tumor cells per slide $(0 \%-100 \%)$ was multiplied by dominant intensity pattern of staining (1, negative or trace; 2 , weak; 3 , moderate; 4 , intense). The overall score ranged from 0 to 400 and specimens were 
considered having levels of EGFR expression: Negative or low (0-200) (considered negative), intermediate (201-300) (Fig. 2A) and high (301400) (Fig. 2B) (intermediate and high considered positive) [17]

Claudin 4: Only membranous staining was classified as positive. Scoring was done according

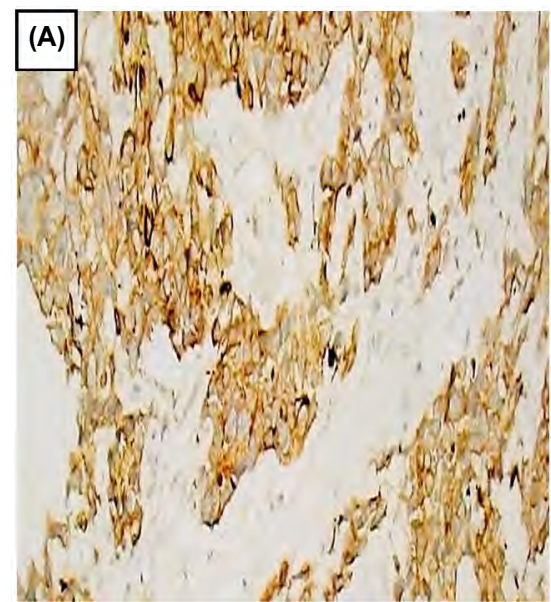

to intensity of expression on a scale of $0-3$ where 0 is negative, 1 is weakly positive, 2 is intermediate positive and 3 is strongly positive. High claudin4 was classified as tumors with a staining intensity of $>2$ (Fig. 3A), and low claudin- 4 was classified as tumors with a staining intensity of $<2$ (Fig. 3B) [18] .

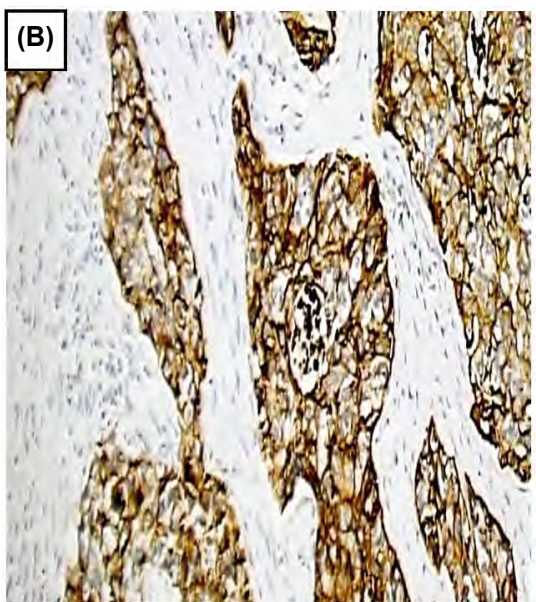

Fig. (1A): IHC to CK5/6, showing moderate cytoplasmic staining score 2 (X200). (B) IHC to CK5/6, showing strong cytoplasmic staining score 3 , (X200).
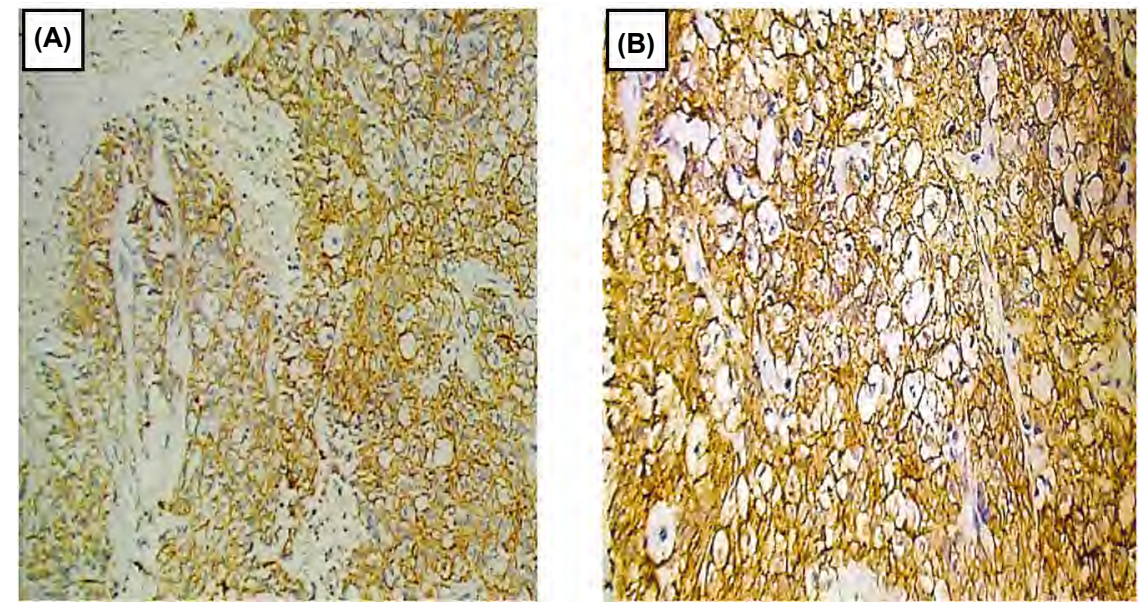

Fig. (2A): IHC to EGFR, showing intermediate membranous staining (x200). (B) IHC to EGFR, showing strong membranous staining (X200).
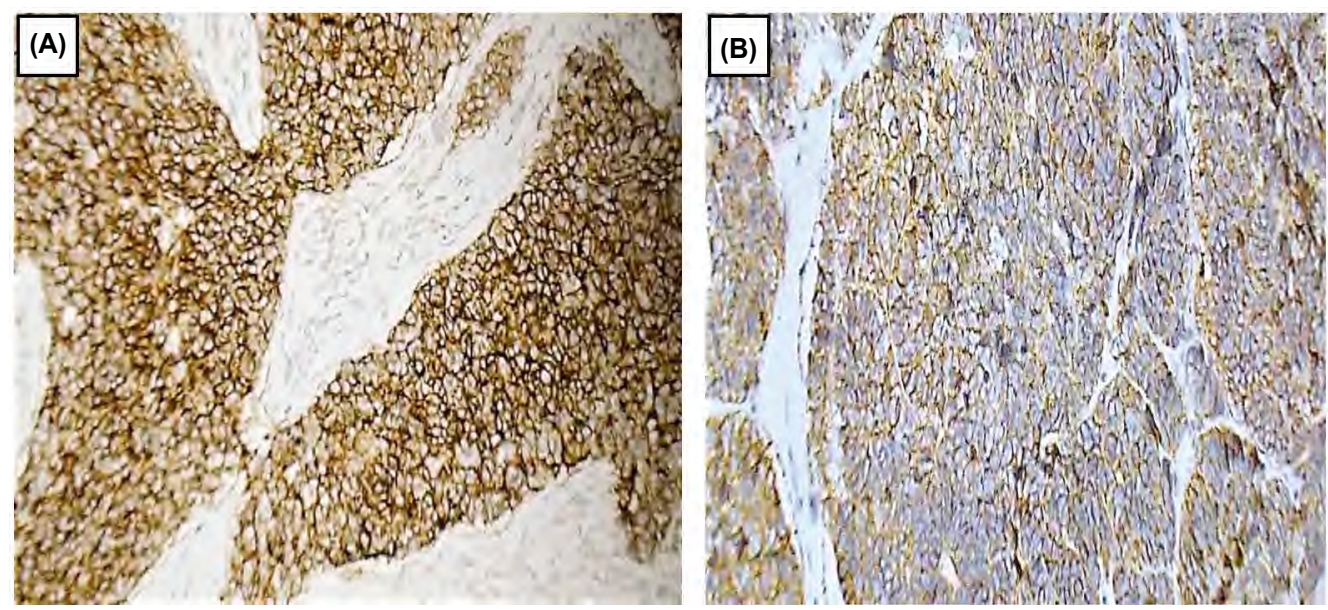

Fig. (3A): IHC to Claudin 4, showing membranous staining, score 3 [high claudin 4] (X200). (B) IHC to Claudin 4 , showing no membranous staining, score 0 [low claudin 4] (X200). 


\section{Statistical methods:}

Data was analyzed using IBM SPSS advanced statistics (Statistical Package for Social Sciences), version 22 (SPSS Inc., Chicago, IL). Numerical data was described as median and interquartile range or range or mean and standard deviation as appropriate, while qualitative data were described as number and percentage. Chi-square (Fisher's exact) test was used to examine the relation between qualitative variables as appropriate.

Survival analysis was done using Kaplan-Meier method. Comparison between two survival curves was done using $\log$ rank test. Multivariate analysis was done by Cox regression model to test for independent prognostic effect of statistically significant variables on univariate level with calculating hazard ratio and its $95 \%$ confidence interval. $p$-value $\leq 0.05$ was considered significant and all test was 2 tailed. Bonferonni corrections of $p$-value was done to avoid hyperinflation of type 1 error which arises from multiple testing.

A $p$-value less than or equal to 0.05 was considered statistically significant. All tests were two tailed.
Overall survival (OS) was calculated from date of diagnosis till date of death or last follows-up.

Disease free survival (PFS) was calculated from date of surgery till date of relapse, death or last follows-up.

\section{Results}

All 59 cases were included in the study were females between the age group 26 to 77 years with the mean age of 48.95 years, and median age was 50 years. All cases were IDC (NST). Most cases were postmenopausal, with negative family history. The majority of cases represented grade 2, T2, stage II, and with positive lymph nodes.

\section{Results of immunohistochemical markers:}

Most of cases were positive to CK5/6 (71.2\%), with majority of cases represented Claudin 4 high $(89.8 \%)$, while (45.8\%) were positive to EGFR.

As regards relation with clinicopathologic parameters, there was a statistically significant relation obtained between CK 5/6 and size of the tumor as well as EGFR and stage of tumor. Relation of immunohistochemical markers and clinicopathologic parameters are listed in (Table 1).

Table (1): Relation of Immunohistochemical markers and clinicopathologic parameters.

\begin{tabular}{|c|c|c|c|c|c|c|c|c|c|}
\hline \multirow{2}{*}{$\begin{array}{l}\text { Clinicopathological } \\
\text { parameters }\end{array}$} & \multicolumn{2}{|c|}{ CK5/6 } & \multirow[b]{2}{*}{$p$-value } & \multicolumn{2}{|c|}{ EGFR } & \multirow[b]{2}{*}{$p$-value } & \multicolumn{2}{|c|}{ Claudin 4} & \multirow[b]{2}{*}{$p$-value } \\
\hline & $\begin{array}{l}\text { Negative } \\
\mathrm{n}(17)\end{array}$ & $\begin{array}{l}\text { Positive } \\
\text { n (42) }\end{array}$ & & $\begin{array}{l}\text { Negative } \\
\text { n (32) }\end{array}$ & $\begin{array}{l}\text { Positive } \\
\text { n (27) }\end{array}$ & & $\begin{array}{l}\text { Negative } \\
n(6)\end{array}$ & $\begin{array}{l}\text { Positive } \\
\text { n (35) }\end{array}$ & \\
\hline $\begin{array}{l}\text { Age }(\text { years }): \\
\quad<=50 \\
>50\end{array}$ & $\begin{array}{l}11(64.7 \%) \\
6(35.3 \%)\end{array}$ & $\begin{array}{l}23(54.8 \%) \\
19(45.2 \%)\end{array}$ & 0.484 & $\begin{array}{l}21(65.6 \%) \\
11(34.4 \%)\end{array}$ & $\begin{array}{l}13(48.1 \%) \\
14(51.9 \%)\end{array}$ & 0.176 & $\begin{array}{l}4(66.7 \%) \\
2(33.3 \%)\end{array}$ & $\begin{array}{l}30(56.6 \%) \\
23(43.4 \%)\end{array}$ & 1.000 \\
\hline $\begin{array}{l}\text { Menopausal status: } \\
\text { Pre } \\
\text { Post }\end{array}$ & $\begin{array}{l}11(64.7 \%) \\
6(35.3 \%)\end{array}$ & $\begin{array}{l}17(40.5 \%) \\
25(59.5 \%)\end{array}$ & 0.091 & $\begin{array}{l}18(56.2 \%) \\
14(43.8 \%)\end{array}$ & $\begin{array}{l}10(37.0 \%) \\
17(63.0 \%)\end{array}$ & 0.141 & $\begin{array}{l}3(50.0 \%) \\
3(50.0 \%)\end{array}$ & $\begin{array}{l}25(47.2 \%) \\
28(52.8 \%)\end{array}$ & 1.000 \\
\hline $\begin{array}{l}\text { Family history: } \\
\text { Positive } \\
\text { Negative }\end{array}$ & $\begin{array}{l}2(11.8 \%) \\
15(88.2 \%)\end{array}$ & $\begin{array}{l}5(11.9 \%) \\
37(88.1 \%)\end{array}$ & 1.000 & $\begin{array}{l}3(9.4 \%) \\
29(90.6 \%)\end{array}$ & $\begin{array}{l}4(14.8 \%) \\
23(85.2 \%)\end{array}$ & 0.692 & $\begin{array}{l}1(16.7 \%) \\
5(83.3 \%)\end{array}$ & $\begin{array}{l}6(11.3 \%) \\
47(88.7 \%)\end{array}$ & 0.701 \\
\hline $\begin{array}{l}\text { Size: } \\
\text { T1\&T2 } \\
\text { T3\&T4 }\end{array}$ & $\begin{array}{l}11(64.7 \%) \\
6(35.3 \%)\end{array}$ & $\begin{array}{l}38(90.5 \%) \\
4(9.5 \%)\end{array}$ & 0.017 & $\begin{array}{l}25(78.1 \%) \\
7(21.9 \%)\end{array}$ & $\begin{array}{l}24(88.9 \%) \\
3(11.1 \%)\end{array}$ & 0.272 & $\begin{array}{l}5(83.3 \%) \\
1(16.7 \%)\end{array}$ & $\begin{array}{l}44(83.0 \%) \\
9(17.0 \%)\end{array}$ & 1.000 \\
\hline $\begin{array}{l}\text { Node status: } \\
\text { Positive } \\
\text { Negative }\end{array}$ & $\begin{array}{l}11(64.7 \%) \\
6(35.3 \%)\end{array}$ & $\begin{array}{l}26(61.9 \%) \\
16(38.1 \%)\end{array}$ & 0.84 & $\begin{array}{l}23(71.8 \%) \\
9(28.2 \%)\end{array}$ & $\begin{array}{l}14(51.8 \%) \\
13(48.2 \%)\end{array}$ & 0.784 & $\begin{array}{l}5(83.4 \%) \\
1(16.6 \%)\end{array}$ & $\begin{array}{l}32(60.4 \%) \\
21(39.6 \%)\end{array}$ & 0.396 \\
\hline $\begin{array}{l}\text { Grade: } \\
\quad 1 \& 2 \\
\quad \text { Grade } 3\end{array}$ & $\begin{array}{l}10(58.8 \%) \\
7(41.2 \%)\end{array}$ & $\begin{array}{l}27(64.3 \%) \\
9(35.7 \%)\end{array}$ & 0.694 & $\begin{array}{l}19(59.4 \%) \\
13(40.6 \%)\end{array}$ & $\begin{array}{l}18(66.7 \%) \\
9(33.3 \%)\end{array}$ & 0.564 & $\begin{array}{l}4(66.7 \%) \\
2(33.3 \%)\end{array}$ & $\begin{array}{l}33(62.3 \%) \\
20(37.7 \%)\end{array}$ & 1.000 \\
\hline $\begin{array}{l}\text { Stage: } \\
1 \& 2 \\
3 \& 4\end{array}$ & $\begin{array}{l}9(52.9 \%) \\
8(47.1 \%)\end{array}$ & $\begin{array}{l}29(69.0 \%) \\
13(31.0 \%)\end{array}$ & 0.242 & $\begin{array}{l}17(53.1 \%) \\
15(46.9 \%)\end{array}$ & $\begin{array}{l}21(77.8 \%) \\
6(22.2 \%)\end{array}$ & 0.049 & $\begin{array}{l}4(66.7 \%) \\
2(33.3 \%)\end{array}$ & $\begin{array}{l}34(64.2 \%) \\
19(35.8 \%)\end{array}$ & 1.000 \\
\hline $\begin{array}{c}\text { Metastasis: } \\
\text { Positive } \\
\text { Negative }\end{array}$ & $\begin{array}{l}3(17.6 \%) \\
14(82.4 \%)\end{array}$ & $\begin{array}{l}6(14.3 \%) \\
36(85.7 \%)\end{array}$ & 0.745 & $\begin{array}{l}6(18.8 \%) \\
26(81.2 \%)\end{array}$ & $\begin{array}{l}3(11.1 \%) \\
24(88.9 \%)\end{array}$ & 0.488 & $\begin{array}{l}1(16.7 \%) \\
5(83.3 \%)\end{array}$ & $\begin{array}{l}8(15.1 \%) \\
45(84.9 \%)\end{array}$ & 0.919 \\
\hline $\begin{array}{l}\text { Recurrence: } \\
\text { Positive } \\
\text { Negative }\end{array}$ & $\begin{array}{l}0(0.0 \%) \\
17(100 \%)\end{array}$ & $\begin{array}{l}5(11.9 \%) \\
37(88.1 \%)\end{array}$ & 0.308 & $\begin{array}{l}3(9.4 \%) \\
29(90.6 \%)\end{array}$ & $\begin{array}{l}2(7.4 \%) \\
25(92.6 \%)\end{array}$ & $*$ & $\begin{array}{l}1(16.7 \%) \\
5(83.3 \%)\end{array}$ & $\begin{array}{l}4(7.5 \%) \\
4(92.5 \%)\end{array}$ & $*$ \\
\hline $\begin{array}{l}\text { Outcome: } \\
\text { Good } \\
\text { Bad }\end{array}$ & $\begin{array}{l}13(76.5 \%) \\
4(23.5 \%)\end{array}$ & $\begin{array}{l}30(71.4 \%) \\
12(28.6 \%)\end{array}$ & 0.693 & $\begin{array}{l}24(75.0 \%) \\
8(25.0 \%)\end{array}$ & $\begin{array}{l}19(70.4 \%) \\
8(29.6 \%)\end{array}$ & 0.690 & $\begin{array}{l}3(50.0 \%) \\
3(50.0 \%)\end{array}$ & $\begin{array}{l}40(75.5 \%) \\
13(24.5 \%)\end{array}$ & 0.330 \\
\hline
\end{tabular}


The relation of positivity of immunohistochemical markers with each other revealed a tendency for cases positive for $\mathrm{CK} 5 / 6$ to be positive also for EGFR, yet this relation did not reach the point of statistical significance.
According to survival analysis, there is a significant relationship found between OS with grade, stage, occurrence of metastasis and node positivity (Table 2) as well as between grade and DFS (Table $3)$.

Table (2): Overall survival and its relation to clinicopathologic parameters and Immunohistochemical markers.

\begin{tabular}{|c|c|c|c|c|c|c|}
\hline & No. & $\begin{array}{l}\text { No. of } \\
\text { events }\end{array}$ & $\begin{array}{c}\text { Cumulative } \\
\text { survival at } 12 \\
\text { months (\%) }\end{array}$ & $\begin{array}{c}\text { Cumulative } \\
\text { survival at } 36 \\
\text { months (\%) }\end{array}$ & $\begin{array}{c}\text { Cumulative } \\
\text { survival at } 60 \\
\text { months (\%) }\end{array}$ & $p$-value \\
\hline Whole group: & 59 & 11 & 96.6 & 84.7 & 79.7 & - \\
\hline \multicolumn{7}{|l|}{ Age: } \\
\hline$\leq 50$ & 34 & 4 & 97.1 & 93.9 & 84.9 & 0.089 \\
\hline$>50$ & 25 & 7 & 92.0 & 72.0 & 72.0 & \\
\hline \multicolumn{7}{|c|}{ Menopausal status: } \\
\hline Pre & 28 & 3 & 100.0 & 96.4 & 85.9 & 0.115 \\
\hline post & 31 & 8 & 93.5 & 74.2 & 74.2 & \\
\hline \multicolumn{7}{|l|}{ Family history: } \\
\hline Positive & 7 & 2 & 85.7 & 71.4 & 71.4 & 0.473 \\
\hline Negative & 52 & 9 & 96.2 & 86.5 & 80.9 & \\
\hline \multicolumn{7}{|l|}{ Grade: } \\
\hline Grade $1 \& 2$ & 37 & 10 & 94.6 & 78.2 & 68.8 & 0.026 \\
\hline Grade 3 & 22 & 1 & 100.0 & 95.5 & 95.5 & \\
\hline \multicolumn{7}{|l|}{ Tumor size: } \\
\hline $\mathrm{T} 1 \& \mathrm{~T} 2$ & 49 & 7 & 95.9 & 87.7 & 84.8 & 0.070 \\
\hline T3 \& T4 & 10 & 4 & 90.0 & 70.0 & 58.3 & \\
\hline \multicolumn{7}{|l|}{ Stage: } \\
\hline Stge $1 \& 2$ & 38 & 9 & 100.0 & 94.7 & 91.2 & 0.003 \\
\hline Stge $3 \& 4$ & 21 & 8 & 90.5 & 66.7 & 60.0 & \\
\hline \multicolumn{7}{|l|}{ LN status: } \\
\hline Positive & 37 & 10 & 94.6 & 78.2 & 69.6 & 0.031 \\
\hline Negative & 22 & 1 & 100.0 & 95.5 & 95.5 & \\
\hline \multicolumn{7}{|l|}{ Metastasis: } \\
\hline Positive & 9 & 6 & 100.0 & 55.6 & NR & $<0.001$ \\
\hline Negative & 50 & 5 & 96.0 & 89.9 & 89.9 & \\
\hline \multicolumn{7}{|l|}{ CK5/6: } \\
\hline Positive & 42 & 8 & 97.6 & 83.3 & 79.4 & 0.905 \\
\hline Negative & 17 & 3 & 94.1 & 88.2 & 80.2 & \\
\hline \multicolumn{7}{|l|}{ EGFR: } \\
\hline Positive & 27 & 4 & 96.3 & 85.2 & 85.2 & 0.603 \\
\hline Negative & 32 & 7 & 96.9 & 84.3 & 76.2 & \\
\hline \multicolumn{7}{|l|}{ Claudin 4: } \\
\hline High & 53 & 9 & 96.2 & 84.8 & 81.6 & 0.408 \\
\hline Low & 6 & 2 & 100.0 & 83.3 & 66.7 & \\
\hline
\end{tabular}

NR: Not reached. 
Table (3): Disease free survival and its relation to clinicopathologic parameters and Immunohistochemical markers.

\begin{tabular}{|c|c|c|c|c|c|c|c|}
\hline & No. & $\begin{array}{l}\text { No. of } \\
\text { events }\end{array}$ & $\begin{array}{c}\text { Cumulative } \\
\text { survival at } 12 \\
\text { months (\%) }\end{array}$ & $\begin{array}{l}\text { Cumulative } \\
\text { survival at } 36 \\
\text { months (\%) }\end{array}$ & $\begin{array}{c}\text { Cumulative } \\
\text { survival at } 60 \\
\text { months (\%) }\end{array}$ & $\begin{array}{l}\text { Median } \\
\text { survival } \\
\text { estimate }\end{array}$ & $p$-value \\
\hline Whole group: & 59 & 20 & 100 & 92.5 & 67.9 & 67.900 & - \\
\hline $\begin{array}{l}\text { Age (years): } \\
\quad \leq 50 \\
\quad>50\end{array}$ & $\begin{array}{l}34 \\
25\end{array}$ & $\begin{array}{l}10 \\
10\end{array}$ & $\begin{array}{l}100 \\
100\end{array}$ & $\begin{array}{l}93.8 \\
95.7\end{array}$ & $\begin{array}{l}82.0 \\
53.8\end{array}$ & $\begin{array}{l}67.900 \\
76.900\end{array}$ & 0.922 \\
\hline $\begin{array}{l}\text { Menopausals } \\
\text { Pre } \\
\text { post }\end{array}$ & $\begin{array}{l}28 \\
31\end{array}$ & $\begin{array}{l}9 \\
11\end{array}$ & $\begin{array}{l}100 \\
100\end{array}$ & $\begin{array}{l}96.2 \\
89.3\end{array}$ & $\begin{array}{l}75.4 \\
61.4\end{array}$ & $\begin{array}{l}66.900 \\
76.900\end{array}$ & 0.764 \\
\hline $\begin{array}{l}\text { Family histor } \\
\text { Positive } \\
\text { Negative }\end{array}$ & $\begin{array}{l}7 \\
52\end{array}$ & $\begin{array}{l}4 \\
16\end{array}$ & $\begin{array}{l}100 \\
100\end{array}$ & $\begin{array}{l}100 \\
91.3\end{array}$ & $\begin{array}{l}75.5 \\
67.0\end{array}$ & $\begin{array}{l}64.900 \\
72.900\end{array}$ & 0.757 \\
\hline $\begin{array}{l}\text { Grade: } \\
\quad \text { Grade1\&2 } \\
\text { Grade } 3\end{array}$ & $\begin{array}{l}37 \\
22\end{array}$ & $\begin{array}{l}17 \\
3\end{array}$ & $\begin{array}{l}100 \\
100\end{array}$ & $\begin{array}{l}88.4 \\
100\end{array}$ & $\begin{array}{l}60.5 \\
85.7\end{array}$ & $\begin{array}{l}66.900 \\
77.900\end{array}$ & 0.035 \\
\hline $\begin{array}{c}\text { Tumor size: } \\
\text { T1\&T2 } \\
\text { T3\&T4 }\end{array}$ & $\begin{array}{l}49 \\
10\end{array}$ & $\begin{array}{l}15 \\
5\end{array}$ & $\begin{array}{l}100 \\
100\end{array}$ & $\begin{array}{l}90.9 \\
100\end{array}$ & $\begin{array}{l}67.2 \\
71.4\end{array}$ & $\begin{array}{l}66.900 \\
75.900\end{array}$ & 0.173 \\
\hline $\begin{array}{l}\text { Stage: } \\
\quad \text { Stge } 1 \& 2 \\
\text { Stge } 3 \& 4\end{array}$ & $\begin{array}{l}38 \\
21\end{array}$ & $\begin{array}{l}11 \\
9\end{array}$ & $\begin{array}{l}97.4 \\
100\end{array}$ & $\begin{array}{l}94.6 \\
89.5\end{array}$ & $\begin{array}{l}69.3 \\
66.1\end{array}$ & $\begin{array}{l}66.900 \\
75.900\end{array}$ & 0.852 \\
\hline $\begin{array}{l}\text { LN status: } \\
\text { Positive } \\
\text { Negative }\end{array}$ & $\begin{array}{l}37 \\
22\end{array}$ & $\begin{array}{l}17 \\
3\end{array}$ & $\begin{array}{l}100 \\
100\end{array}$ & $\begin{array}{l}91.1 \\
95.5\end{array}$ & $\begin{array}{l}65.6 \\
27.7\end{array}$ & $\begin{array}{l}66.900 \\
*\end{array}$ & 0.276 \\
\hline $\begin{array}{l}\text { CK5/6: } \\
\text { Positive } \\
\text { Negative }\end{array}$ & $\begin{array}{l}42 \\
17\end{array}$ & $\begin{array}{l}14 \\
6\end{array}$ & $\begin{array}{l}100 \\
100\end{array}$ & $\begin{array}{l}92.0 \\
93.8\end{array}$ & $\begin{array}{l}71.9 \\
57.3\end{array}$ & $\begin{array}{l}72.900 \\
66.900\end{array}$ & 0.795 \\
\hline $\begin{array}{l}\text { EGFR: } \\
\text { Positive } \\
\text { Negative }\end{array}$ & $\begin{array}{l}27 \\
32\end{array}$ & $\begin{array}{l}10 \\
10\end{array}$ & $\begin{array}{l}100 \\
100\end{array}$ & $\begin{array}{l}98.5 \\
89.5\end{array}$ & $\begin{array}{l}60 \\
76.9\end{array}$ & $\begin{array}{l}64.900 \\
75.900\end{array}$ & 0.261 \\
\hline $\begin{array}{l}\text { Claudin 4: } \\
\text { High } \\
\text { Low }\end{array}$ & $\begin{array}{l}53 \\
6\end{array}$ & $\begin{array}{l}17 \\
3\end{array}$ & $\begin{array}{l}100 \\
100\end{array}$ & $\begin{array}{l}93.7 \\
83.3\end{array}$ & $\begin{array}{l}68.6 \\
62.5\end{array}$ & $\begin{array}{l}64.900 \\
72.900\end{array}$ & 0.200 \\
\hline
\end{tabular}

\section{Discussion}

Many studies reveled that triple negative breast cancer is a heterogeneous group of breast cancer [19]. Basal markers such as CK5/6 and EGFR as well as Claudin 4 are considered important in subclasssification of the triple negative group and can also affect treatment options [20,21].

In our study most of cases positive to CK5/6 were $<$ or equal 50 years. Cases expressed CK5/6 were characterized by T2 size ( $71.4 \%)$, showing statistically significant relationship between expression of CK5/6 and tumor size ( $p$-value 0.017). Most of the positive cases are of grade II (64.3\%), followed by grade III ( $35.7 \%)$ and none is of grade I. The greatest percentage of node positive status is among positive group (61.9\%). This aggressive course was reflected on the stage of the CK 5/6 positive cases with stage 2 represented $(61.9 \%)$ followed by stage $3(28.5 \%)$, and the only case of stage 4 , belonged to the CK $5 / 6$ positive group, with all the cases who underwent recurrence and 6/9 cases who underwent metastasis are CK5/6 positive. Similar findings were reported by Preethi and colleagues, with range of age at presentation among CK 5/6 positive patients was $41-58$ years, commonest size was $\mathrm{T} 2$, commonest grade is grade $2(57.2 \%)$ followed by grade $3(42.8 \%)$, and $50 \%$ showed node metastasis [16]. Nearly similar results were also reported in other studies [22]

As regards EGFR (45.8\%) represented positive cases. The relation of EGFR with clinicopathologic parameters revealed that stage II was the commonest stage, with significant relationship with stage and expression of EGFR ( $p$-value 0.049). Most of cases positive to EGFR were more than 50 years, of grade II tumors $(66.7 \%)$ of T2 $(77.8 \%)$, and with node positivity $(51.8 \%)$. Only three cases underwent metastasis, and two underwent recurrence, with no significant relationships detected with any of these parameters. 
Discrepant results are retrieved from various studies as regards prognostic impact of EGFR on TNBC. Some studies agreed with ours as Sobande and colleagues who reported no significant relationship between expression of EGFR with grade and node positivity denoting that EGFR changes did not appear to be associated with markers of aggressive behavior in TNBC [23]. Others reported bad prognosis of EGFR positive cases [22]

To reach a definite conclusion about prognostic impact of the expression of EGFR a lot of studies with large sample size are intensively needed, to justify the routine implication of this marker for stratification of patients with TNBC and to identify candidates who could benefit from its targeted therapy.

Minority of cases represented Claudin low group which were only six cases $(10.2 \%)$, which is similar to many studies denoted that Claudin low group revealed minor cases among TNBC $[24,25]$

Claudin high type was reported in $89.8 \%$ of our cases. Most cases were of age $<=50$. Cases of $\mathrm{T} 2$, T3, and all cases of T4, most high grades (2\&3), high stages, and node positivity were all Claudin high, however, none of these parameters reached statistically significant relation. Eight cases underwent metastasis from total nine in this study and four cases underwent recurrence from total five are of Claudin high group. Abd-Elazeem and Abd-Elazeem who studied also Egyptian patients reported similar figures as most of cases of Claudin high were less than 50 years, showed tumor size $\mathrm{T} 2$ and T3, high grade, high stage, and node positivity [26]. Also similar results were reported by Ko and colleagues [27]. These results point to that high expression of Claudin 4 may be associated with poor prognosis and would provide a strong prognostic power to differentiate the patients with worse outcome among triple negative breast cancer patients. Kolokytha and colleagues reported that increased immunohistochemical expression of Claudin-4 was found to be related to favorable prognostic parameters among triple negative but not luminal type breast cancer [10]. This highlights that expression of the same protein could exert different prognostic impact among different molecular subtypes of breast cancer. Also ethnic factors may play role in different impact.

Although Claudins act to increase cell to cell adhesions and thus expected for tumors over expressing them to behave in a less aggressive man- ner, our results are actually the opposite. An assumption that among triple negative molecular subtype, different signaling pathways could interfere with post-translational modification of the expressed Claudin 4, could explain this controversy [10] More trails and studies concerning Claudin 4 and its relation to different molecular subtypes are needed to clarify the mechanism of action of this protein and the credibility of the administration of targeted therapy with the use of anticlaudin antibodies.

A significant relationship was found between the disease free and overall survival with grade of the tumor, which revealed the effectiveness of the chemotherapy treatment on the higher grade tumor with improvement of the survival, with similarity to many studies [28]

Finally, we concluded that among Egyptian patients, triple negative breast cancer did not comprise one disease group. It could be better stratified according to the expression of different immunohistochemical markers which could help in decision making as regards enrollment in clinical trials and application of new therapeutic agents targeting the different expressed proteins by the tumor. Also, the different results we obtained as regard prognostic impact of EGFR and Claudin 4 from other literature is an interesting and encouraging point to further study these markers on Egyptian population and clarify if there is statistically significant difference which could be attributed to ethnic factors, also concluded that the well established traditional factors which are the clinicopathologic parameters still play the most crucial role in prognosis, other than the markers which need more studies on large scale of cases.

\section{References}

1- SIEGEL L., MILLER D. and JEMAL A.: Cancer statistics. Ca. Cancer J., 67: 7-30, 2017.

2- MOHAMED G.: Breast cancer In: Mokhtar N., Salama A., Badawy O., Khorshed E., Abdelazim H.: Cancer Pathology Registry 2000-2011; Chapter 2, pp 8-3 1, 2016.

3- RAKHA E. and REIS-FILHO J.S.: Basal-like breast carcinoma: From expression profiling to routine practice. Archives of pathology \& laboratory medicine, 133 (6): 860-868, 2009.

4- PEROU C.M., SØRLIE T., EISEN M.B., VAN DE RIJN M., JEFFREY S.S., REES C. A., et al.: Molecular portraits of human breast tumours. Nature, 406 (6797): 747.-752, 2000 .

5- NIELSEN T.O., HSU F.D., JENSEN K., CHEANG M., KARACA G., HU Z., et al.: Immunohistochemical and clinical characterization of the basal-like subtype of 
invasive breast carcinoma. Clinical Cancer Research, 10 (16): 5367-5374, 2004.

6- CAREY L.A., PEROU C.M., LIVASY C.A., et al.: Race, breast cancer subtypes, and survival in the Carolina Breast Cancer Study. JAMA, 295: 2492-2, 2006.

7- TELLI M.L., CHANG E.T., KURIAN A.W., KEEGAN T.H., MCCLURE L.A., LICHTENSZTAJN D., et al.: Asian ethnicity and breast cancer subtypes: A study from the California Cancer Registry. Breast Cancer Research and Treatment, 127 (2): 471-478, 2011.

8- ALSHAREEDA A.T., SORIA D., GARIBALDI J.M., RAKHA E., NOLAN C., ELLIS I.O. and GREEN A.R.: Characteristics of basal cytokeratin expression in breast cancer. Breast Cancer Research and Treatment, 139 (1): 23-37, 2013.

9- BOUCHALOVA K., CIZKOVA M., CWIERTKA K., TROJANEC R. and HAJDUCH M.: Triple Negative Breast Cancer-Current Status And Prospective Targeted Treatment Based On Her1 (EGFR), Top2a and C-Myc Gene Assessment. Biomedical Papers of the Medical Faculty of Palacky University in Olomouc, 153: 13-8, 2009.

10- KOLOKYTHA P., YIANNOU P., KERAMOPOULOS D., KOLOKYTHAS A., NONNI A., PATSOURIS E. and PAVLAKIS K.: Claudin-3 and claudin-4: Distinct prognostic significance in triple-negative and luminal breast cancer. Applied Immunohistochemistry \& Molecular Morphology, 22 (2): 125-131, 2014.

11- BHALLA A., MANJARI M., KAHLON S.K., KUMAR P. and KALRA N.: Cytokeratin 5/6 expression in benign and malignant breast lesions. Indian. J. Pathol. Microbiol., 53: 676, 2010.

12-FADARE O., WANG S.A. and HILEETO D.: The expression of cytokeratin 5/6 in invasive lobular carcinoma of the breast: evidence of a "basal-like" subset? Human Pathology, 39 (3): 331-336, 2008.

13- LAKHANI S.R., ELLIS I.O., SCHNITT S.J., TAN P.H. and VAN de VIJVER M.J.: Tumors of the breast In World Health Organization Classification of Tumors. Pathology and Genetics of tumors of breast and female genital organs, 4th Edition, p8. IARC Press: Lyon, 2012.

14- EASTON D.F.: Familial risks of breast cancer. Breast Cancer Research, 4 (5): 179, 2002.

15- EDGE S.B. and COMPTON C.C.: The American Joint Committee on Cancer: the $7 \mathrm{t}^{\mathrm{h}}$ edition of the AJCC cancer staging manual and the future of TNM. Annals of Surgical Oncology, 17 (6): 1471-147, 2010.

16- PREETHI S., AIL D.A. and PRAKASH G.: Study of CK5/6 expression in invasive breast carcinomas and its utility as an independent prognostic marker. Global Journal for Research Analysis, 6 (1): 99-102, 2017.

17- GORI S., SIDONI A., COLOZZA M., FERRI I., MAMELI M.G., FENOCCHIO D., et al.: EGFR, pMAPK, pAkt and PTEN status by immunohistochemistry: correlation with clinical outcome in HER2-positive metastatic breast cancer patients treated with trastuzumab. Annals of Oncology, 20 (4): 648-654, 2009.

18- LANIGAN F., MCKIERNAN E., BRENNAN D.J., HEGARTY S., MILLIKAN R.C., MCBRYAN J., et al.: Increased claudin-4 expression is associated with poor prognosis and high tumour grade in breast cancer. InterNational Journal of Cancer, 124 (9): 2088-2097, 2009.

19- AHN S.G., LEE H.M., LEE H.W., LEE S.A., LEE S.R., LEEM, S.H., et al.: Prognostic discrimination using a 70gene signature among patients with estrogen receptorpositive breast cancer and an intermediate 21 -gene recurrence score. International Journal of Molecular Sciences, 14 (12): 23685-23699, 2016.

20- TAN D.S., MARCHIÓ C., JONES R.L., SAVAGE K., SMITH I. E., DOWSETT M. and REIS-FILHO J.S.: Triple negative breast cancer: Molecular profiling and prognostic impact in adjuvant anthracycline-treated patients. Breast cancer research and treatment, 111 (1): 27 44, 2008.

21- THIKE A.A., CHEOK P.Y., JARA-LAZARO A.R., TAN B., TAN P. and TAN P.H.: Triple-negative breast cancer: Clinic-pathological characteristics and relationship with basal-like breast cancer. Modern Pathology, 23 (1): 123 133, 2010.

22- SOOD N. and NIGAM J.S.: Correlation of CK5 and EGFR with clinicopathological profile of triple-negative breast cancer. Pathology Research International, 2014.

23- SOBANDE F., DUSEK L., MATEJKOVÁ A., ROZKOS T., LACO J. and RYSKA A.: EGFR in triple negative breast carcinoma: Significance of protein expression and high gene copy number. Cesk Patol., 51 (2): 80-86, 2015.

24- CHOI J., JUNG W.H. and KOO J.S.: Clinicopathologic features of molecular subtypes of triple negative breast cancer based on immunohistochemical markers. Histology and histopathology, 27 (10): 1481, 2012.

25- SABATIER R., FINETTI P., GUILLE A., ADELAIDE J., CHAFFANET M., VIENS P., et al.: Claudin-low breast cancers: Clinical, pathological, molecular and prognostic characterization. Molecular Cancer, 13 (1): 228, 2014.

26- ABD-ELAZEEM M.A. and ABD-ELAZEEM M.A.: Claudin 4 expression in triple-negative breast cancer: Correlation with androgen receptors and Ki-67 expression. Annals of Diagnostic Pathology, 19 (1): 37-42, 2015.

27- KO B.S., KIM H.J., YU J.H., LEE J.W., SOHN B.H., KIM S.B., et al.: Claudin 1, 3, 4, and 7 expressions in triple-negative breast cancer. J. Clin. Oncol., 1070, 2013.

28- PINDER S.E., MURRAY S., ELLIS I.O., TRIHIA H., ELSTON C.W., GELBER R.D., et al.: The importance of the histologic grade of invasive breast carcinoma and response to chemotherapy. Cancer, 83 (8): 1529-1539, 1998. 


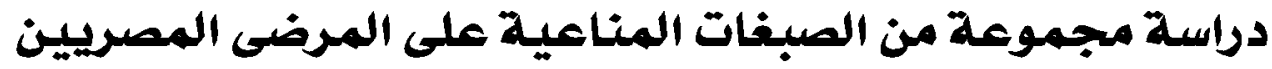

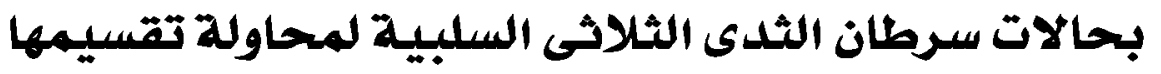

سرطان الثدى الثلاثى السلبية من مجموعات سرطان الثدى التى تخضع لعدة دراسات لمحاولة تقسيمها إلى مجموعات صغيرة

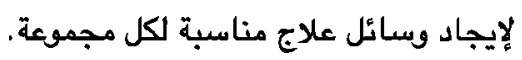

الهدف من الدراسة: دراسة وأجراء مجموعة من الصبغات المناعية على حالات سرطان الثدى الثلاثى السلبية لمحاولة تقسيمها

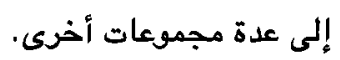

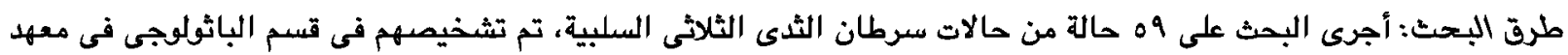

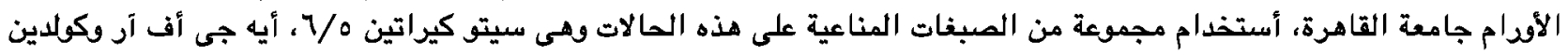

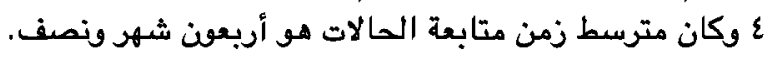

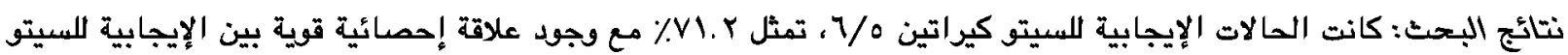

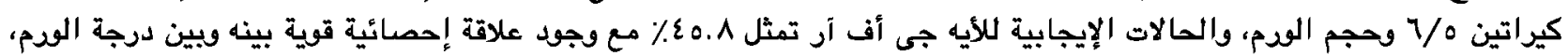

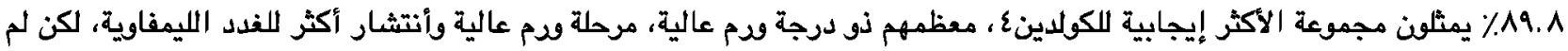

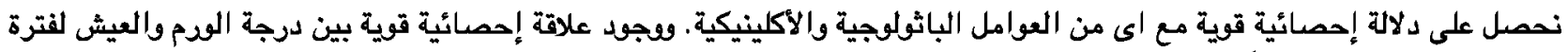

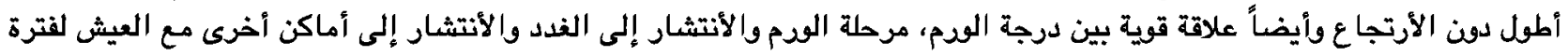

الأستتاج: مجموعة سرطان الثدى الثلاثى السلبية مجموعة مختلطة تحتاج إلى مجموعة من الصبغات المناعية مثل سيتو كيراتين

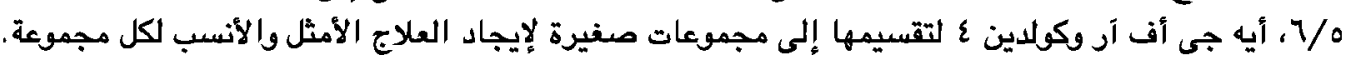

